\title{
Relationships between compulsive exercise, quality of life, psychological distress and motivation to change in adults with anorexia nervosa
}

Sarah Young ${ }^{1 *}$, Stephen Touyz ${ }^{2}$, Caroline Meyer ${ }^{3}$, Jon Arcelus ${ }^{4,5}$, Paul Rhodes $^{2}$, Sloane Madden ${ }^{6,7}$, Kathleen Pike ${ }^{8}$, Evelyn Attia ${ }^{9}$, Ross D. Crosby ${ }^{10,11}$ and Phillipa Hay ${ }^{12}$ D

\begin{abstract}
Background: For people with anorexia nervosa (AN), compulsive exercise is characterized by extreme concerns about the perceived negative consequences of stopping/reducing exercise, dysregulation of affect, and inflexible exercise routines. It is associated with increased eating disorder psychopathology and poor clinical outcome. However, its relationships with two important clinical issues, quality of life (QoL) and motivation to change, are currently unknown. This study aimed to assess the cross-sectional relationships between compulsive exercise, QoL, psychological distress (anxiety and depressive symptoms, and obsessive-compulsive traits) and motivation to change in patients with AN.
\end{abstract}

Method: A total of 78 adults with AN participated in this study, which was nested within a randomized controlled trial of psychological treatments for AN. At baseline (pre-treatment), participants completed questionnaires assessing compulsive exercise, eating disorder (ED) psychopathology, QoL, psychological distress and motivation to change.

Results: Baseline correlational analyses demonstrated a moderate positive relationship between compulsive exercise and ED psychopathology, and a weak positive relationship between compulsive exercise and psychological distress. There was a moderate negative relationship between compulsive exercise and eating disorder QoL.

Conclusions: These results indicate compulsive exercise is moderately associated with poorer QoL and weakly associated with higher distress. Targeting compulsive exercise in the treatment of anorexia nervosa may help reduce the burden of illness and improve patients' engagement in treatment.

Trial registration: ACTRN12610000585022. Taking a LEAP forward in the treatment of anorexia nervosa: a randomized controlled trial. NHMRC grant: 634922.

Keywords: Compulsive exercise, Anorexia nervosa, Quality of life, Obsessive-compulsive, Anxiety, Depression, Motivation to change

\footnotetext{
* Correspondence: syou5950@uni.sydney.edu.au

${ }^{1}$ Griffith Taylor Building, School of Psychology, University of Sydney, Sydney,

Australia

Full list of author information is available at the end of the article
} 


\section{Plain English summary}

Many people with anorexia nervosa (AN) engage in compulsive exercise as a part of their eating disorder. This type of exercise can be defined as very driven and rigid exercise behaviours and attitudes. Although compulsive exercise has been linked to poorer physical and mental health outcomes, little is known about how it impacts on an individual's quality of life, and their motivation to change their eating disorder. We conducted a study with 78 adults with AN (including 4 males) who were taking part in a larger eating disorder treatment study. Before they started treatment, participants completed questionnaires which asked about eating disorder symptoms, compulsive exercise, quality of life, symptoms of anxiety, depression and obsessivecompulsive features, and their motivation to change. Our results found that people who exercised compulsively experienced more severe eating disorder symptoms. They also demonstrated poorer quality of life relating to their eating disorder and more elevated symptoms of depression and anxiety. Focusing on compulsive exercise in treatment for AN may help patients to improve their quality of life and mood.

\section{Background}

Anorexia nervosa (AN) is associated with a variety of negative physical, psychological and psychosocial effects [1], as well as functional impairment and reduced quality of life (QOL) [2, 3]. Adding to the illness burden are common co-morbidities such as obsessive-compulsive disorder (OCD) and/or obsessive-compulsive traits as well as low levels of treatment engagement [4, 5]. An important aspect of AN psychopathology, given scarce attention in the QoL and treatment engagement literature, is compulsive exercise. This is lacking despite the high prevalence of problematic exercise, its association with compulsivity, and detrimental effects on treatment and illness course [6-8].

Compulsive exercise can be described as an individual's extremely driven and inflexible exercise patterns, together with a perceived lack of capacity to stop exercising, despite awareness of its negative effects [9]. It has been associated with higher levels of psychological distress in $\mathrm{AN}$, including greater depressive and anxious traits. A conceptual model of compulsive exercise [10] emphasizes both the positively and negatively reinforcing contributions of this type of exercise on affect regulation. Although patients exercise to improve their mood, compulsive exercise can also be maintained by the individual to reduce high levels of negative emotions, primarily anxiety $[11,12]$. It is often performed to avoid withdrawal symptoms such as irritability and low mood. These symptoms can occur when an individual is unable to exercise due to treatment restrictions/recommendations $[13,14]$.

In community samples, two characteristics of exercise have been significantly associated with lower QoL and higher ED psychopathology: (1) feeling guilty when unable to exercise; and (2) exercising predominantly to change weight or shape [15-17]. These findings in the community underline the need to explore associations between compulsive exercise, QoL and ED psychopathology in patients with $\mathrm{AN}$.

Another important facet of AN which can impact upon treatment is the presence of obsessive-compulsive traits. These are a common comorbidity of AN [18] and are closely related to the concept of compulsivity, a key maintaining factor in Meyer et al.'s cognitive-behavioural model of exercise [10]. A positive association between problematic exercise in $\mathrm{AN}$ and obsessive-compulsive personality disorder (OCPD) traits has been demonstrated [19]. In contrast, studies of the relationship between obsessive-compulsive disorder (OCD) and excessive exercise have been inconsistent [19]. It may be that examining obsessive-compulsive traits could be more relevant in patients with AN. An updated conceptualization of compulsive exercise and a corresponding self-report measure have since been developed [9] and this relationship can now be examined more closely.

Low motivation to change can also have a significant impact upon progress in treatment for $\mathrm{AN}$, and has been associated with lower BMI [20], slower weight gain [21] and poorer overall QoL [22]. However, little is known about the relationship between compulsive exercise and motivation to change. As compulsive exercise has been associated with other indicators of eating disorder severity such as being a predictor of relapse [23] and having longer hospitalizations [24], it would be interesting to assess whether there is an association between exercise and motivation to change before treatment. Many patients with AN endorse compulsive exercise driven by shape and weight concerns, and as a method of affect regulation [10]. This is an area we wish to explore as it can be argued that these characteristics could contribute to reduced motivation to change. The trans-theoretical model of motivation to change [25] has been applied to patients with AN and posits that patients (with various illness diagnoses) can move between 6 stages of change ranging from pre-contemplation to maintenance and termination.

In summary, compulsive exercise is a core feature of $\mathrm{AN}$, which can add great complexity to the course of the illness and have a negative impact upon treatment $[6,12]$. Furthermore, exercise is associated with greater eating disorder psychopathology and higher levels of depression and anxiety. The relationship between compulsive exercise and QoL has been demonstrated in non-clinical studies 
but needs to be replicated in the clinical population. Little is known about the relationships between compulsive exercise and QOL, and between compulsive exercise and motivation to change in patients with AN. As obsessivecompulsive traits can compromise treatment outcome, the relationship between exercise in $\mathrm{AN}$ and these traits also requires further examination.

The current study thus aimed to assess the relationships between severity of compulsive exercise with subjective ratings of eating disorder psychopathology, QOL, psychological distress and motivation to change pre-treatment. It was hypothesized that compulsive exercise would be negatively related to QOL, but positively related to psychological distress. Given the paucity of previous research investigating compulsive exercise and motivation to change, no specific hypothesis was made for this relationship.

\section{Method}

\section{Participants}

A total of 78 adults (including 4 males) participated in the current study, all of whom were enrolled in a multisite randomized controlled trial (RCT) "Taking a LEAP forward in the treatment of anorexia nervosa" [26]. The trial took place in Leicester, United Kingdom; Sydney, Australia; and New York, United States of America. The main aim of the parent trial was to examine a novel cognitive-behavioural therapy module for compulsive exercise (compuLsive Exercise Activity theraPy or LEAP) [27], and to determine if it improved treatment outcomes for patients with AN who exercise. Participants were randomly allocated into one of two groups. The first group completed 34 sessions of mCBT-AN [28], a cognitivebehavioural therapy (CBT) which aims to normalize eating and help patients reach and maintain a healthy weight. The second group had eight sessions of LEAP treatment, and 26 sessions of mCBT-AN. LEAP aims to provide psychoeducation to patients about distinctions between healthy/balanced and compulsive exercise, utilizing CBT techniques to manage unhelpful exercise behaviours and beliefs [27]. A pilot study of LEAP for inpatients with AN has demonstrated its acceptability [29].

All participants were recruited for the trial through referral from an eating disorder clinic/service, or from public advertising. Potential participants had to be 18 years or older to enrol in the trial, have a DSM-5 primary diagnosis of AN [30] using the Eating Disorder Examination (EDE) interview [31], and have a Body Mass Index (BMI) between 14 and 18.5. Participants were assessed for medical stability and this was monitored regularly throughout the trial. To enrol in the trial, participants had to have participated in exercise in the previous month, as indicated on the Exercise Participation Screening Questionnaire (participated in an activity on at least one occasion in the previous 4 weeks) or scored at least one time in the past month on the Eating Disorder Examination Questionnaire, i.e. self-report that he/she participated in at least one occasion of exercise in the past 4 weeks (EDE-Q) [32]. This criterion was broad as the study aimed to recruit participants performing a range of exercise behaviour, but participants did not need to view their exercise as compulsive. Exclusion criteria were a diagnosis of psychosis or bipolar disorder, current substance dependence, high suicidality, medical instability and concurrent treatment for their eating disorder. There were 574 potential participants who expressed interest in the trial. However, 496 were deemed ineligible or did not wish to participate, hence there were 78 who were enrolled and randomized in the trial.

Ethics was approved at each site: the Western Sydney University Human Research Ethics Committee; National Health Service Research Ethics Committee in the UK, as part of the Health Research Authority; and the Institutional Review Board at Columbia University in New York, USA. Participants gave written informed consent for the treatment trial and for completion of research assessments.

The mean age of the participants at initial assessment in the current study was 27.38 years (SD: 9.22, median: 24.7, interquartile range: $20.1-31.0$ years), their mean BMI was 16.52 (SD: 1.12, median: 16.8, interquartile range: 15.7-17.5) and the average duration of illness (from diagnosis of AN to enrolment in the trial) was 5.65 years (SD: 7.88, median:1.40; interquartile range: .6-8.5 years). $71.8 \%$ met criteria for AN-Restrictive subtype, whilst $28.2 \%$ met criteria for AN Binge-eating/ Purging subtype. Of the participants, $32.1 \%$ were born in Australia, 50\% were born in the UK, $11.5 \%$ in the USA, and the remaining $6.4 \%$ were born elsewhere. A total of $52.5 \%$ of the sample had completed university, whilst a further $35.9 \%$ had completed at least 10 years of schooling. At the time of the study, $57.7 \%$ worked full-time or part-time, $26.9 \%$ were students, $12.8 \%$ were unemployed and $2.6 \%$ completed full-time house duties. Most participants were single (69.3\%), 26.9\% were married or living in long-term relationships, and $3.8 \%$ were separated or divorced.

\section{Measures}

Participants completed self-report questionnaires assessing ED psychopathology, compulsive exercise, psychological distress, motivation to change and quality of life. These questionnaires were presented to participants in the order below.

Eating Disorder Examination-Questionnaire (EDE-Q) [32] is a 36-item questionnaire, comprised of the four subscales in the EDE interview [31]. It has demonstrated good psychometrics in clinical samples [33]. Participants completed the full EDE-Q, but only the EDE-Q global score was used in the analyses. Higher scores indicate 
greater eating disorder psychopathology. The Cronbach's $\alpha$ for the EDE-Q global score in the current study was .94 .

Short Form-12 Health Status Questionnaire (SF-12) [34] assesses quality of life by investigating the functional limitations related to physical and mental health conditions. It consists of 12 Likert-style response items that examine a range of domains including Physical Functioning e.g. "Does your health now limit you in these activities? Moderate activities, such as moving a table, pushing a vacuum cleaner, bowling or playing golf"; Bodily Pain e.g. "During the past four weeks, how much did pain interfere with your normal work, including both work outside the home and housework?"; and Social functioning e.g. "During the past four weeks, how much of the time has your physical health or emotional problems interfered with your social activities like visiting friends or relatives?" This study used two subscales, the Physical Health Component Summary Scale (PCS), and the Mental Health Component Summary Scale (MCS). Higher scores indicate a greater level of functioning. The SF-12 has previously demonstrated good psychometric properties [34] and the Cronbach's $\alpha$ for the overall scale was .89. The SF-12 can compare QoL across a variety of illness categories [35, 36].

Kessler-10 item distress scale (K-10) [37] is a measure of psychological distress (operationalized as anxiety and depression symptoms), originally developed as a screening tool for these symptoms in community samples. The 10 -item questionnaire includes questions such as "Over the past four weeks (28 days), how often have you felt so nervous that nothing could calm you down?" and "Over the past four weeks (28 days), how often have you felt that everything was an effort?". Higher scores (maximum score of 50) indicate greater psychological distress, with large community surveys by the Australian Bureau of Statistics classifying scores of $30-50$ to be in the Very High distress range [38]. It is a valid measure for patients with eating disorders [39]. The total score was used in the current study, and the Cronbach's $\alpha$ was 92 .

Padua Inventory (Padua) [40] is a 39-item measure of psychological distress, specifically assessing obsessivecompulsive traits. It consists of six subscales: Contamination obsessions and washing compulsions e.g. "I feel my hands are dirty when I touch money"; Dressing/Grooming compulsions e.g. "I feel obliged to follow a particular order in dressing, undressing, and washing myself"; Checking compulsions e.g. "I have to do things several times before I think they are properly done"; Obsessional thoughts of harm to self/others e.g. "I think or worry at length about having hurt someone without knowing it"; and Obsessional impulses to harm self/others e.g. "When I see a train approaching, I sometimes think I could throw myself under its wheels". It has demonstrated good psychometric properties [41] and is valid for use in clinical samples. Higher scores indicate greater obsessive-compulsive traits. The mean total score was used in the current study and the Cronbach's $\alpha$ for the scale was 93 .

Eating Disorder Quality of Life (EDQoL) [42] is a 25-item measure of eating disorders-specific quality of life with four subscales: Psychological "How often has your eating/weight made you feel worse about yourself?"; Physical/Cognitive "How often has your eating/weight affected your ability to pay attention when you wanted to?"; Work/School "How often has your eating/weight led to a leave of absence from work?"; and Financial "How often has your eating/weight led to the need to spend money from savings or use your credit card frequently?". These are summed for a mean total score, with higher scores representing lower quality of life. The EDQoL has been previously used in patients with eating disorders [36] and patients with chronic AN [35]. The Cronbach's $\alpha$ for the overall scale was .93. ED-specific QOL measures can be used to compare results of different treatment interventions $[35,36]$.

The Anorexia Nervosa Stages of Change Questionnaire (ANSOCQ) [43] is a validated measure of motivation to change in patients with AN. It consists of 20 items, assessing three factors of Weight Gain (readiness to achieve a minimum healthy body weight), Eating, shape and weight concerns (readiness to change shape and weight importance in defining achievement and satisfaction in life), and Ego-alien aspects (readiness to change interpersonal issues associated with AN). Each item has 5 answers representing the different stages of change: pre-contemplation; contemplation; preparation; action; and maintenance. The participant marks the response/s that is most in line with their current attitudes. Example items include Weight gain (pre-contemplation response) "As far as I am concerned I do not need to gain weight"; Eating, shape and weight concerns (action response) "I often try to challenge the importance that I place on my body shape or weight in determining my happiness and success"; and Ego-alien aspects (maintenance response) "The problems in my relationships with others have improved and I am trying to keep it this way". Higher scores indicate greater motivation to change. The following mean scores correspond to the different stages of change: $<1.5=$ pre-contemplation; $1.5-2.4=$ contemplation; $2.5-3.4=$ preparation; $3.5-4.4=$ action; $>4.5=$ maintenance . Cronbach's $\alpha$ for the scale was .91 .

Compulsive Exercise Test (CET) [9] is a 24-item selfreport questionnaire examining the core maintaining factors of compulsive exercise for patients with eating disorders. It consists of 5 subscales: Avoidance and ruledriven behavior (CET-Avoidance, e.g. "I usually continue to exercise despite injury or illness, unless I am very ill or too injured"); Weight control exercise (CET-Weight 
control, e.g. "If I feel I have eaten too much, I will do more exercise"); Mood improvement (CET-Mood, e.g. "I feel less stressed and/or tense after I exercise"); Lack of exercise enjoyment (CET-Lack of enjoyment, e.g. "I find exercise a chore") and Exercise rigidity (CET-Rigidity, e.g. "I like my days to be organized and structured, of which exercise is just one part"). The CET-Total score is used in this study, calculated by summing the means for the five subscales. Higher scores reflect more compulsive exercise. It is psychometrically sound and has been validated for use in adult clinical samples [44, 45]. A clinical cut-off score of 15 is effective in differentiating between compulsive and non-compulsive exercise in patients with diagnosed eating disorders [44]. In the current study, only the CET-Total was used, and its Cronbach's $\alpha$ was .92.

\section{Data analysis}

The Shapiro-Wilk test demonstrated that the data were not normally distributed for the EDE-Q, Padua Inventory, ANSOCQ and CET, thus non-parametric tests (Spearman's rho) were used in the analyses. Due to hypotheses, onetailed tests were used, except for the ANSOCQ which used a two-tailed test. The significance level was set at .05 and analyses were performed using SPSS, Version 22.

\section{Results}

\section{Correlations between compulsive exercise, QOL, distress and motivation to change}

The aim of this study was to examine the relationships between compulsive exercise, QOL, psychological distress and motivation to change prior to treatment (see Table 1). CET-Total was positively and moderately associated with higher eating disorder psychopathology (EDE-Q). CET-Total was not significantly associated with the SF-12 Mental health Component score, but it showed a weak negative association with the SF-12 Physical health Component Score, meaning poorer physical health quality of life. CET-Total was moderately associated with higher EDQOL scores, indicating poorer quality of life.
Our results demonstrated a weak positive association between CET-Total and psychological distress, as measured by both the Kessler-10 and the Padua Inventory. There was no significant association between CET-total and motivation to change (ANSOCQ overall stage score).

\section{Discussion}

The current study aimed to assess the relationships of compulsive exercise to QOL, psychological distress and motivation to change pre-treatment. Confirming the hypotheses, baseline correlations demonstrated weak positive associations between compulsive exercise (CET-Total) and psychological distress (anxiety and mood symptoms on the K-10), aligning with previous research [11, 46]. There was also a weak positive relationship between exercise and obsessive-compulsive traits, confirming previous results $[8,47,48]$. However, this contrasted Bewell-Weiss and Carter's study [49], which also used the Padua Inventory and found excessive exercise to be negatively associated with obsessive-compulsive symptomatology. Their study utilized a quantitative definition of compulsive exercise and categorized patients as excessive exercisers if they endorsed obligatory exercise for at least $1 \mathrm{~h}$ per day, on at least 6 days per week for 1 month. Our use of the CET examines the cognitive-behavioural characteristics of compulsive exercise, rather than the obligatory and quantitative aspects of compulsive exercise which may account for our different results.

A novel aspect of the current study was the examination of the relationships between compulsive exercise and quality of life. At baseline, compulsive exercise was moderately associated with poorer ED-related quality of life (EDQoL) and weakly associated with lower general physical-health quality of life (SF-12 PCS). There are few studies reporting upon these relationships in a clinical sample of outpatients, and our results emphasize the detrimental impact exercise can have upon patients' general functioning. Mond and Calogero [50] found that the two factors which differentiated between eating disorder patients and healthy women were the same as those associated with poorer quality of life in community samples, i.e. exercising for shape and weight reasons, and avoidance of guilt if exercise was missed [15-17]. However, they did

Table 1 Spearman's correlations between compulsive exercise and variables of interest at baseline

\begin{tabular}{lllll}
\hline Measure $(N=78)$ & $M$ & SD & CET-Total $(M=16.26, S D=4.39)$ & $p$ \\
\hline Eating Disorder Examination-Questionnaire (EDE-Q) & 3.99 & 1.40 & $.41^{* *}$ & $-.21^{*}$ \\
SF-12 Physical health Component Score (SF-12 PCS) & 46.74 & 10.43 & -.18 & .001 \\
SF-12 Mental health Component Score (SF-12 MCS) & 29.32 & 11.83 & $.23^{*}$ & .05 \\
Kessler-10 (K-10) & 31.04 & 9.54 & $.23^{*}$ & .02 \\
Padua Inventory (Padua) & 23.68 & 19.52 & $.36^{* *}$ & .02 \\
Eating Disorder Quality of Life (EDQOL) & 1.69 & .68 & .02 \\
Anorexia Nervosa Stages of Change Questionnaire (ANSOCQ) & 2.61 & .63 & .05 \\
\hline
\end{tabular}

${ }^{*}=p<.05,{ }^{* *}=p<.01$ 
not specifically investigate quality of life in this study. Other research examining an Australian sample of inpatients with EDs found a significant positive relationship between QOL and driven exercise [51]. Their study used the Quality of Life-Eating Disorders measure (QOL-ED) from the computer-generated Eating and Exercise Examination (EEE-C) [52] but looked only at the frequency of exercise and did not directly assess the compulsive qualities of exercise.

As compulsive exercise is associated with a wide range of physical health complaints [8], it was expected that there would be a significant relationship between compulsive exercise and poorer physical health QoL (measured by the PCS on the SF-12) prior to treatment. However, an unexpected finding was that the association between compulsive exercise and the mental-health component of the SF-12 (MCS) approached significance but was not significant $(p=.05)$. The MCS measures the functional impairment from emotional issues upon work and social activities. Patients may experience their exercise to be adaptive, in that the behavior serves as both a way to improve their mood and to avoid/manage feelings of guilt and depression if they are unable to exercise. This could have impacted upon their ratings of functional impairment. Although the SF-12 has been evaluated as a sensitive measure of changes in ED pathology [35], it may also be that the MCS is not as sensitive to the positively and negatively reinforcing functions of compulsive exercise behavior.

Compulsive exercise was not significantly associated with lower motivation to change (ANSOCQ) in this pretreatment sample $(p=.055)$. However, we know that patients with a lower level of motivation for change demonstrate a greater rigidity of ED beliefs and are more ambivalent about reducing their ED behaviours. Given these negative characteristics of the illness are associated with poorer motivation to change, it is integral that therapists and medical specialists accurately assess patients' motivation to change. Future research should investigate this relationship longitudinally across treatment, as it may be that focusing on and addressing exercise in treatment [53] is more important for those who demonstrate lower motivation to change.

There were several strengths in the current study, namely that it incorporated both ED specific and general health QOL measures, as this has been recommended in recent reviews in the field $[35,36]$. This study is novel in that no previous research has specifically investigated the relationships between compulsive exercise, quality of life and motivation to change in a clinical sample of outpatients with AN. It did include a small group of male patients with AN, but unfortunately it was not possible to conduct analyses based on gender due to our sample size. Men may experience more functional impairment than women from some ED symptoms e.g. objective binge eating [54], thus it would be beneficial for future studies to utilize larger samples of males with clinical EDs.

This study utilized a range of quality of life measures, however another eating disorder specific measure of HRQoL, e.g. Clinical Impairment Assessment (CIA) [55] may have been a useful addition. As our participants were medically stable and seen on a regular basis as outpatients, our results may not generalize to inpatients or day program patients. Although most of the correlations between compulsive exercise and variables of interest were significant, some of the correlations were weak in magnitude, e.g. K-10, Padua and SF-12 PCS. Finally, our patients were enrolled in an RCT addressing compulsive exercise and it was a criterion of entry that they had participated in at least one form of physical activity in the past month. These low exercise inclusion criteria were used to generalize as best as possible to patients with AN who engage in exercise (of any frequency), however it is important to acknowledge that these results may not generalize to all patients with AN. Despite having a low entry threshold for exercise, the mean CET score in this sample still fell above the clinical cut off score of 15 indicated for patients with AN who are compulsive exercisers [44].

\section{Conclusions}

This study demonstrated that greater compulsive exercise is moderately associated with poorer ED quality of life, and higher levels of ED psychopathology. Greater compulsive exercise is also weakly associated with higher levels of psychological distress. Addressing compulsive exercise is integral to reducing the burden of illness from AN and may be important in improving people's engagement in treatment. Further research is required to investigate the relationship between these variables, and to utilize longitudinal designs to assess if severity of compulsive exercise predicts these factors over time and throughout treatment.

\section{Abbreviations \\ AN: Anorexia Nervosa; ANSOCQ: Anorexia Nervosa Stages of Change Questionnaire; BMI: Body Mass Index; CBT: Cognitive-Behavioural Therapy; CE: Compulsive Exercise; CET: Compulsive Exercise Test; DSM-5: Diagnostic and Statistical Manual of Mental Disorders 5th edition; ED: Eating Disorder; EDE-Q: Eating Disorder Examination-Questionnaire; EDQoL: Eating Disorder Quality of Life scale; K-10: Kessler 10-item Distress Scale; \\ LEAP: Loughborough Eating disorders Activity theraPy; MCS: Mental Health Component Summary Scale; OCD: Obsessive-Compulsive Disorder; OCPD: Obsessive-Compulsive Personality Disorder; PCS: Physical Health Component Summary Scale; PI: Padua Inventory; QOL: Quality of Life; RCT: Randomized Controlled Trial; SF-12: Short Form-12 Health Status Questionnaire}

\section{Acknowledgements}

The authors would like to sincerely thank Andreea Heriseanu, Jackie Wales, Jessica Lerman and Lauren Belak for their work as research coordinators for the LEAP trial. 


\section{Funding}

This study was supported by an NHMRC Project Grant no. 634922. HAY, Prof Phillipa; TOUYZ, Prof Stephen; MEYER, Dr. Caroline; ARCELUS, Dr. Jon; MADDEN Dr. Sloane; CROSBY, Dr. Ross; PIKE, Dr. Kathleen. Taking a LEAP forward in the treatment of Anorexia Nervosa: A randomized controlled trial \$557,100, 20102013. SY was supported by an Australian Postgraduate Award scholarship.

\section{Availability of data and materials}

The datasets analysed during the current study are available from the corresponding author on reasonable request.

\section{Authors' contributions}

This study was nested within the RCT "Taking a LEAP forward in the treatment of anorexia nervosa". PH, ST, CM, JA, SM, RDC, KP and EA are chief investigators of this trial. SY developed the study idea and designed the study with PH and ST. RDC assisted SY with statistical analyses and interpretation of data. SY prepared the manuscript and all authors contributed to and approved the final manuscript.

\section{Ethics approval}

Ethics approval was granted by the Western Sydney University Human Research Ethics Committee; the National Health Service Research Ethics Committee in the UK, as part of the Health Research Authority; and the Institutional Review Board at Columbia University in New York, USA.

\section{Consent for publication}

Not applicable as the project did not report individual data.

\section{Competing interests}

ST and PH receive royalties from Routledge, Hogrefe, and Huber and McGraw-Hill publishers for printed contributions on eating disorders. SM and ST receive honoraria from Shire Pharmaceuticals. PH received royalties from Oxford University Press and receives sessional fees and lecture fees from the Australian Medical Council, Therapeutic Guidelines publication, and New South Wales Institute of Psychiatry. RDC is a statistical consultant to Health Outcome Solutions, in Winter Park, Florida. PH and ST are Editors-in-Chief of this journal, and CM, JA, SM, KP, JA, RDC and SY are on the editorial board. All authors have other published papers cited in this manuscript. SY submitted an alternate version of this manuscript as a part of her PhD thesis.

\section{Publisher's Note}

Springer Nature remains neutral with regard to jurisdictional claims in published maps and institutional affiliations.

\section{Author details}

Griffith Taylor Building, School of Psychology, University of Sydney, Sydney, Australia. ${ }^{2}$ School of Psychology, University of Sydney, Sydney, Australia. ${ }^{3}$ WMG, University of Warwick, United Kingdom \& University Hospitals Coventry and Warwickshire NHS Trust, Coventry, UK. ${ }^{4}$ Institute of Mental Health, Faculty of Medicine \& Health Sciences, University of Nottingham, Nottingham, UK. ${ }^{5}$ Leicestershire Adult Eating Disorders Service, Leicestershire Partnership NHS Trust, Bennion Centre, Glenfield Hospital, Leicester, UK. ${ }^{6}$ School of Medicine, University of Sydney, Sydney, Australia. ${ }^{7}$ Eating Disorders Service at the Sydney Children's Hospitals Network, Westmead, Australia. ${ }^{8}$ Division of Behavioral Health Services and Policy Research, Columbia University, New York, USA. ${ }^{9}$ Department of Psychiatry, College of Physicians and Surgeons of Columbia University, Weill Cornell Medical College, New York, USA. ${ }^{10}$ Neuropsychiatric Research Institute, Fargo, North Dakota, USA. ${ }^{11}$ University of North Dakota School of Medicine and Health Sciences, Fargo, North Dakota, USA. ${ }^{12}$ Translational Health Research Institute (THRI), School of Medicine, Western Sydney University, Campbelltown, Australia.

Received: 12 August 2017 Accepted: 25 January 2018 Published online: 05 February 2018

\section{References}

1. Zipfel S, Giel KE, Bulik CM, Hay P, Schmidt U. Anorexia nervosa: aetiology, assessment, and treatment. Lancet Psych. 2015;2:1099-111.

2. Winkler LA, Christiansen E, Lichtenstein MB, Hansen NB, Bilenberg N, Støving RK. Quality of life in eating disorders: a meta-analysis. Psych Res. 2014;219:1-9.
3. Mitchison D, Hay P, Mond J, Slewa-Younan S. Self-reported history of anorexia nervosa and current quality of life: findings from a community-based study. Qual Life Res. 2013;22:273-81.

4. Keski-Rahkonen A, Hoek HW, Susser ES, Linna MS, Sihvola E, Raevuori A, et al. Epidemiology and course of anorexia nervosa in the community. Am J Psych. 2007:164:1259-65.

5. Hart LM, Granillo MT, Jorm AF, Paxton SJ. Unmet need for treatment in the disorders: a systematic review of eating disorder specific treatment seeking among community cases. Clin Psych Rev. 2011;31:727-35.

6. Noetel M, Dawson L, Hay P, Touyz S. The assessment and treatment of unhealthy exercise in adolescents with anorexia nervosa: a Delphi study to synthesize clinical knowledge. Int J Eat Disord. 2017:50:378-88.

7. Dalle Grave R, Calugi S, Marchesini G. Compulsive exercise to control shape or weight in eating disorders: prevalence, associated features and treatment outcome. Comp Psych. 2008:49:346-52.

8. Shroff H, Reba L, Thornton LM, Tozzi F, Klump KL, Berrettini WH, et al. Features associated with excessive exercise in women with eating disorders. Int J Eat Disord. 2006;39:454-61.

9. Taranis L, Touyz S, Meyer C. Disordered eating and exercise: development and preliminary validation of the compulsive exercise test (CET). Eur Eat Dis Rev. 2011;19:256-68.

10. Meyer C, Taranis L, Goodwin H, Haycraft E. Compulsive exercise and eating disorders. Eur Eat Disord Rev. 2011;19:174-89.

11. Holtkamp K, Hebebrand J, Herpertz-Dahlmann B. The contribution of anxiety and food restriction on physical activity levels in acute anorexia nervosa. Int J Eat Disord. 2004;36:163-71.

12. Noetel M, Miskovic-Wheatley J, Costa D, Crosby RD, Hay P, Kohn M, et al. Exercise for the compulsive exercisers? An exploratory study in adolescent inpatients with anorexia nervosa. Adv Eat Disord. 2016;4:3.

13. Bamber D, Cockerill IM, Rodgers S, Carroll D. Diagnostic criteria for exercise dependence in women. Br J Sports Med. 2003;37:393-400.

14. Hausenblas HA, Symons DD. Exercise dependence: a systematic review. J Sport Exerc Psychol. 2002;3:89-123.

15. Mond JM, Rodgers B, Hay PJ, Owen C, Beumont PJV. Relationships between exercise behaviour, eating- disordered behaviour and quality of life in a community sample: when is exercise "excessive"? Eur Eat Disord Rev. 2004; 12:265-72.

16. Mond JM, Hay PJ, Rodgers B, Owen C, Beumont PJV. An update on the definition of "excessive exercise" in eating disorders research. Int J Eat Disord. 2006;39:147-53.

17. Mond JM, Myers TC, Crosby RD, Hay P, Mitchell J. Excessive exercise and eating-disordered behaviour in young adult women: further evidence from a primary care sample. Eur Eat Disord Rev. 2008;16:215-21.

18. Swinbourne J, Touyz SW. The comorbidity of eating disorders and anxiety disorders: a review. Eur Eat Disord Rev. 2007:15:253-74.

19. Young S, Rhodes P, Touyz S, Hay P. The relationship between obsessivecompulsive personality disorder traits, obsessive-compulsive disorder and excessive exercise in patients with anorexia nervosa: a systematic review. J Eat Disord. 2013;1:16.

20. Geller J, Cassin SE, Brown KE, Srikameswaran S. Factors associated with improvements in readiness for change: low vs normal BMl eating disorders. Int J Eat Disord. 2004;42:40-6.

21. Schebendach JE, Mayer LE, Devlin MJ, Attia E, Contento IR, Wolf RL, Walsh BT. Dietary energy density and diet variety as predictors of outcome in anorexia nervosa. Am J Clin Nutrit. 2008:87:810-6.

22. Munoz PM, Quintana JM, Las Hayas C, Padierna A, Aguirre U, Gonzalez-Torres MA. Quality of life and motivation to change in eating disorders: perception of patient psychiatrist. Eat Behav. 2012;13:131-4

23. Carter JC, Blackmore E, Sutander-Pinnock K, Woodside DB. Relapse in anorexia nervosa: a survival analysis. Psych Med. 2004;34:671-9.

24. Solenberger S. Exercise and eating disorders: a 3-year inpatient hospital record analysis. Eat Behav. 2001:2:151-68.

25. Prochaska JO, DiClemente CC, Norcross JC. In search of how people change: applications to addictive behaviors. Am Psych. 1992;47:1102-14.

26. Hay PJ, Touyz S, Meyer CM, Arcelus J, Crosby RD, Pike K, et al. A randomized controlled trial of the Loughborough eating disorders activity therapy (LEAP): a new approach to driven exercise in anorexia nervosa. Presented at the 21st annual meeting of the Eating Disorders Research Society 2016, New York, October 29

27. Taranis L, Meyer C, Touyz S, Arcelus J, La Puma M. Loughborough eating disorders activity Programme "LEAP". Individual cognitive-behavioural 
therapy for compulsive exercise in the eating disorders: therapist manual. Loughborough: Loughborough University Centre for Research into Eating Disorders (LUCRED); 2011.

28. Pike KM, Walsh BT, Vitousek K, Wilson GT, Bauer J. Cognitive behavior therapy in the post-hospitalization treatment of anorexia nervosa. Am J Psych. 2003;160:2046-9.

29. La Puma M, Touyz S, Taranis L, Meyer C. Group cognitive-Behavioural therapy for excessive exercise in the eating disorders. Fremantle: Proceedings of the 6th annual Australian and New Zealand Academy for Eating Disorders (ANZAED) Conference; 2008.

30. American Psychiatric Association. Diagnostic and statistical manual of mental disorders (5 $5^{\text {th }}$ ed.). Washington: American Psychiatric Association; 2013.

31. Fairburn CG, Cooper Z, O'Connor M. The eating disorders examination. In: Fairburn CG, editor. Cognitive behavior therapy and eating disorders. New York: Guildford Press; 2008. p. 265-308.

32. Fairburn CG, Beglin SJ. Assessment of eating disorders: interview or selfreport questionnaire? Int J Eat Disord. 1994;16:363-70.

33. Berg KC, Peterson CB, Frazier P, Crow SJ. Psychometric evaluation of the eating disorder examination and eating disorder examination-questionnaire: a systematic review of the literature. Int J Eat Disord. 2012;45:428-38.

34. Ware JE, Kosinski M, Keller SD. A 12-item short-form health survey: construction of scales and preliminary tests of reliability and validity. Med Care. 1996:34:220-33.

35. Mitchison D, Hay P, Engel S, Crosby RD, Le Grange D, Lacey H, et al. Assessment of quality of life in people with severe and enduring anorexia nervosa: a comparison of generic and specific instruments. BMC Psych. 2013;13:284.

36. Ackard D, Richter S, Egan A, Engel S, Cronemeyer CL. The meaning of (quality of) life in patients with eating disorders: a comparison of generic and disease-specific measures across diagnosis and outcomes. Int J Eat Disord. 2014;47:259-67.

37. Kessler RC, Andrews G, Colpe L, Hiripi E, Mroczek DK, Normand SL, et al. Short screening scales to monitor population prevalences and trends in non-specific psychological distress. Psych Med. 2002;32:959-76.

38. Australian Bureau of Statistics (ABS). 4817.0.55.001 - Information Paper: Use of the Kessler-10 Psychological Distress Scale in ABS Health Surveys, Australia, 2007-08. Canberra: Author.

39. Mond JM, Hay PJ, Rodgers B, Owen C, Beumont PJV. Assessing quality of life in eating disorder patients. Qual Life Res. 2005;14:171-8.

40. Burns GL, Keortge S, Formea G, Sternberger L. Revision of the Padua inventory of obsessive- compulsive disorder symptoms: distinctions between worry, obsessions, and compulsions. Beh Res Ther. 1996;34:163-73.

41. Sternberger LG, Burns GL. Obsessions and compulsions: psychometric properties of the Padua inventory with an American college population. Beh Res Ther. 1990;28:341-5.

42. Engel SG, Wittrock DA, Crosby RD, Wonderlich SA, Mitchell JE, Kolotkin RL. Development and psychometric validation of an eating-disorder specific health-related quality of life instrument. Int J Eat Disord. 2006;39:62-71.

43. Rieger E, Touyz S, Schotte D, Beumont P, Russell J, Clarke S, et al. Development of an instrument to assess readiness to recover in anorexia nervosa. Int J Eat Disord. 2000;28:387-96.

44. Meyer C, Plateau CR, Taranis L, Brewin N, Wales J, Arcelus J. The compulsive exercise test: confirmatory factor analysis and links with eating psychopathology among women with clinical eating disorders. J Eat Disord. 2016:4:22.

45. Young S, Touyz S, Meyer C, Arcelus J, Rhodes P, Madden S, et al. Validity of exercise measures in adults with anorexia nervosa: the EDE, compulsive exercise test and other self-report scales. Int J Eat Disord. 2017;50:533-41.

46. Penas-Lledo E, Vaz Leal FJ, Waller G. Excessive exercise in anorexia nervosa and bulimia nervosa: relation to eating characteristics and general psychopathology. Int J Eat Disord. 2002;31:370-5.

47. Davis C, Kaptein S. Anorexia nervosa with excessive exercise: a phenotype with close links to obsessive-compulsive disorder. Psych Res. 2006;142:209-17.

48. Naylor H, Mountford V, Brown G. Beliefs about excessive exercise in eating disorders: the role of obsessions and compulsions. Eur Eat Disord Rev. 2011; 19:226-36.

49. Bewell-Weiss CV, Carter JC. Predictors of excessive exercise in anorexia nervosa. Comp Psych. 2010;51:566-71.

50. Mond JM, Calogero R. Excessive exercise in eating disorder patients and in healthy women. ANZ J Psych. 2009;43:227-34.
51. Abraham SF, Brown T, Boyd C, Luscombe G, Russell J. Quality of life: eating disorders. ANZ J Psych. 2006;40:150-5.

52. Abraham SF, Lovell N. Research and clinical assessment of eating and exercise behaviour. Hosp Med. 1999;60:481-5.

53. Beumont PJV, Arthur B, Russell JD, Touyz SW. Excessive physical activity in dieting disorder patients: proposals for a supervised exercise program. Int J Eat Disord. 1994;15:21-36.

54. Mitchison D, Mond J, Slewa-Younan S, Hay P. Sex differences in healthrelated quality of life impairment associated with eating disorder features: a general population study. Int J Eat Disord. 2013;46:375-80.

55. Bohn K, Fairburn CG. Clinical Impairment Assessment questionnaire (CIA 3. 0). In: Fairburn CG, editor. Cognitive behavior therapy and eating disorders. New York: Guildford Press; 2008. p. 315-7.

\section{Submit your next manuscript to BioMed Central and we will help you at every step:}

- We accept pre-submission inquiries

- Our selector tool helps you to find the most relevant journal

- We provide round the clock customer support

- Convenient online submission

- Thorough peer review

- Inclusion in PubMed and all major indexing services

- Maximum visibility for your research

Submit your manuscript at www.biomedcentral.com/submit
Biomed Central 